\title{
Identification of Niclosamide as a New Lead Compound to Suppress the Metastasis of Prostate Cancer Cells
}

\section{Xiaomei Ren, Zhang Zhang, Lei Duan, and Ke Ding*}

Institute of Chemical Biology, Guangzhou Institute of Biomedicine and Health, Chinese Academy of Sciences, \#190 Kaiyuan Avenue, Guangzhou Science Park, Guangzhou, China

\begin{abstract}
Prostate cancer is one of the most common cancer types among males. Metastasis in advanced stages of prostate cancer makes the disease refractory to current treatments and is the leading cause of death of advanced prostate cancer. Drugs targeting metastasis may provide new therapeutic strategies for advanced prostate cancer. We identified the traditional antihelminthic drug niclosamide to have great potential in suppressing the metastatic process of prostate cancer. The drug potently inhibited the migration and invasion of the DU145 prostate cancer cell line. It significantly suppressed the protein levels of key members of the matrix metalloproteinase family (MMP2 and MMP9), as well as cadherins and catenins (E-/N-cadherin and $\beta$-catenin), all of which are critical factors in the metastastic process. Further investigation suggested that the motility inhibition of niclosamide on DU145 cells was highly correlated to its suppression of the STAT3 and MAPK signaling pathways.
\end{abstract}

Graphical Abstract

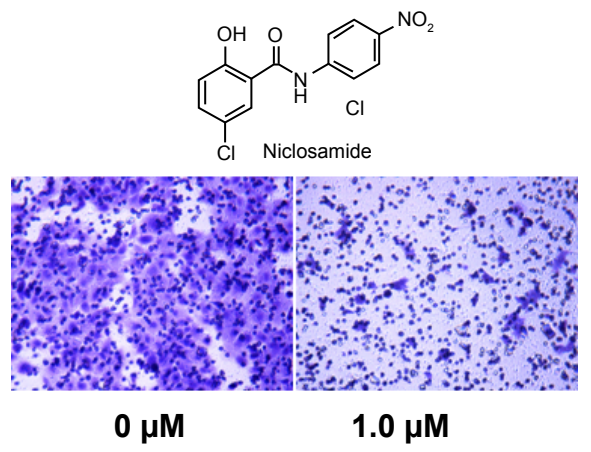

Keywords: Prostate Cancer; Metastasis; Niclosamide; STAT3; MAPK

Prostate cancer (PCA) is one of the most-frequently diagnosed cancers in men and the second leading cause of cancer-related deaths in the United States [1,2]. Although PCA can be treated with surgery and androgen-deprivation based hormonal therapy during early stages $[3,4]$, the disease eventually progresses to an advanced stage where it becomes refractory to treatment and incurable with current therapies [5]. Metastasis of the primary tumor to distant sites is a major cause of the morbidity and mortality in advanced PCA, and it becomes a challenge in clinical management of the disease [6]. For instance, metastasis of PCA to the bone is observed in $\sim 80 \%$ of prostate cancer patients [7]. Therefore, new approaches are needed that not only suppress the proliferation of the primary PCA but also prevent the cancer from progressing to a metastatic state.

Although numerous efforts have been made to illustrate the molecular events leading to the origin and progression of prostate tumors, the mechanisms underlying tumorigenesis and metastasis of the disease are poorly understood. Only a handful of factors have been identified as correlated to PCA, including the Signal transducer and activator of transcription 3 (STAT3) and Mitogen activated protein kinase (MAPK).

STAT3 is a member of the STAT family and is a key transcription factor that regulates various fundamental cellular and biological processes. The STAT3 pathway has recently been recognized as a new oncogenic pathway $[8,9]$ in addition to the Ras/MAPK and PI3/Akt pathways. Constitutively activated STAT3 has been observed in PCA as well as in many other types of malignant tumors [10,11]. Inhibiting the STAT3 pathway has proven to suppress the growth of PCA $[12,13]$. Moreover, STAT3 signaling has recently been shown to be related to the metastatic process of PCA. Upregulated STAT3 expression has been found to contribute to the dysregulation of the motility and invasiveness of prostate cancer cells [14-16].

MAPK has also been associated with PCA $[17,18]$, and recently, the MAPK pathway was proven to be crucial for the metastasis of PCA. Inactivation of MAPK signaling led to decreased migration and invasion of PCA $[19,20]$.

Given the critical roles of STAT3 and MAPK in the tumorigenesis and metastasis of PCA, targeting STAT3 and MAPK signaling pathways might provide a novel therapeutic strategy for the treatment of both primary and metastatic prostate cancers.

*Corresponding author: Ke Ding, Institute of Chemical Biology, Guangzhou Institute of Biomedicine and Health, Chinese Academy of Sciences, \#190 Kaiyuan Avenue, Guangzhou Science Park, Guangzhou, China, Tel: 86-20-32015276; E-mail: ding_ke@gibh.ac.cn

Received March 24, 2014; Accepted April 25, 2014; Published April 28, 2014

Citation: Ren X, Zhang Z, Duan L, Ding K (2014) Identification of Niclosamide as a New Lead Compound to Suppress the Metastasis of Prostate Cancer Cells. Med chem 4: 511-516. doi:10.4172/2161-0444.1000187

Copyright: (c) 2014 Ren X, et al. This is an open-access article distributed under the terms of the Creative Commons Attribution License, which permits unrestricted use, distribution, and reproduction in any medium, provided the original author and source are credited. 
We have recently reported that niclosamide, a classic anthelmintic drug, exerts a potent inhibitory effect on the STAT3 signaling pathway and the proliferation of various cancer cells expressing constitutively active STAT3, including the DU145 prostate cancer cell line [21]. In the present study, we report that niclosamide could efficiently inhibit the motility of DU145 cells by decreasing their migration and invasion.

The effect of niclosamide on the motility of DU145 cells were firstly evaluated by assessing the migratory and invasive properties of the cells. DU145 cells were chosen as the experimental model because of their high motility rate and their constitutively active STAT3 status.

In the wound healing assay, the wound of control cells was obviously healed at 24 hours, and a completed wound closure was observed at 48 hours after initiation, reflecting the strong migratory ability of the DU145 cells. However, niclosamide treatment significantly inhibited the wound healing process in DU145 cells in a dose-dependent manner (Figure 1A). The drug displayed significant inhibition on the wound closure by $\sim 20 \%, \sim 60 \%$ and $\sim 70 \%$ at the concentrations of $0.2,1.0$ and $5.0 \mu \mathrm{M}(\mathrm{P}<0.05,0.01$ and 0.01$)$, respectively (Figure $1 \mathrm{C})$. The inhibitory effect became more evident at 48 hours after the wounds were created.
A complete wound closure was observed in the untreated group whereas wounds remained obviously visible in all of the niclosamidetreated groups.

The effect of niclosamide on the invasion ability of DU145 cells was investigated using a Boyden chamber assay. This assay is commonly used to mimic the in vivo invasion process of cancer cells. ECM was coated on the filter membrane and the number of DU145 cells that were able to invade through the ECM to the other side of the membrane was quantified. Niclosamide remarkably inhibited the invasion of DU145 cells in a concentration-dependent manner (Figure 1B). Treatment with niclosamide at $0.1,0.5$, and $2.0 \mu \mathrm{M}$ for 24 hours inhibited invasion by $\sim 40 \%, \sim 80 \%$ and $\sim 90 \%$, respectively ( $\mathrm{P}<0.01$, compared to the vehicle control) (Figure 1D). These results strongly suggested that niclosamide potently inhibits both migration and invasion of DU145 cells and niclosamide may potentially suppress the metastatic process of prostate cancer.

Degradation of the ECM is one of the most critical steps for promoting metastasis [22-24]. MMP2 and MMP9 are key enzymes mediating the ECM degradation process. Increased levels of MMP2

A niclosamide (uM)

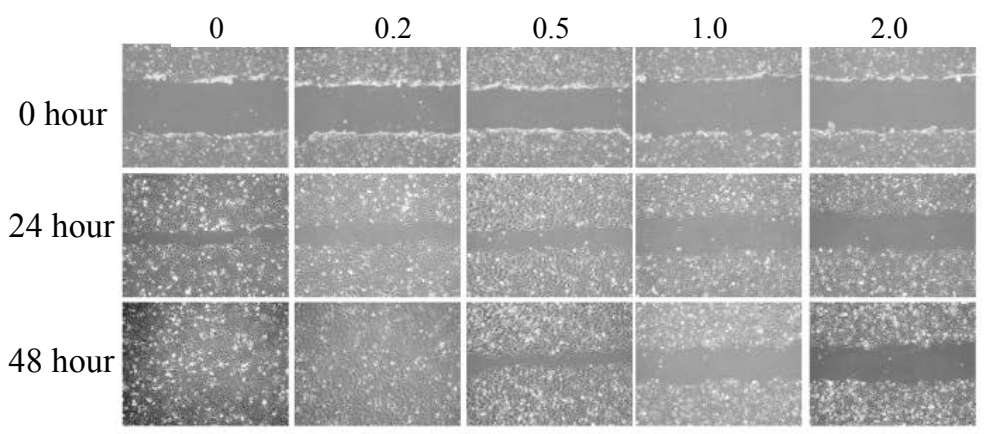

B

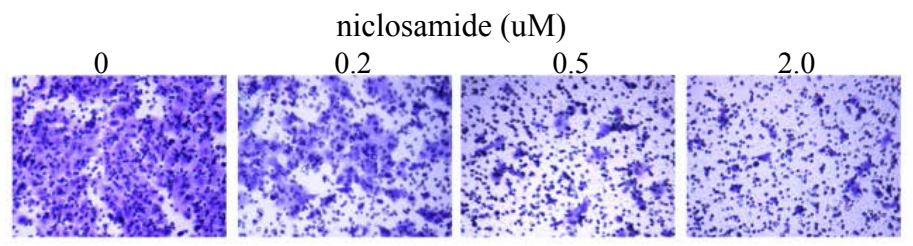

C

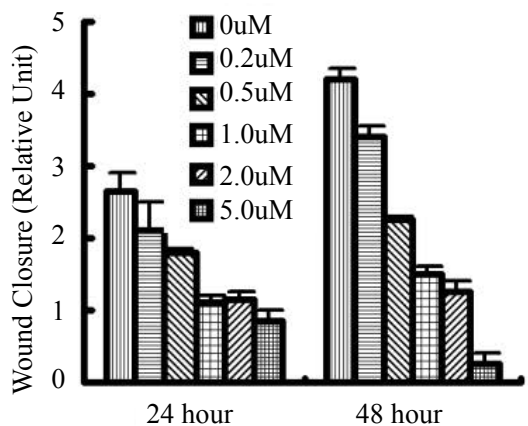

D

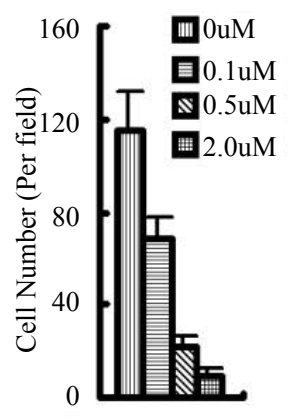

Figure 1: Niclosamide inhibits the motility of DU145 cells. A: Inhibition of niclosamide on migration of DU145 cells. Cells were treated with niclosamide at the indicated concentrations after the wounds were created. Images were taken at the indicated time points. B: Inhibition of niclosamide on invasion of DU145 cells. DU145 cells were plated on an ECM-coated Boyden chamber and treated with niclosamide at the indicated concentrations. Invading cells were photographed 24 hours later. $\mathbf{C}$ : Quantification of A. D: Quantification of B. The results are representative of three independent experiments. 
and MMP9 have been associated with the metastasis of different types of solid tumors [25-28]. Meanwhile, cadherins and their associated catenin family members have been identified as the key factors involved in the endothelial mesenchymal transition (EMT), which is closely related to the progression and metastasis of cancer. Abnormal expression of cadherins and catenins is closely related to an increased incidence of tumor metastasis [29-32].

To achieve a better understanding of the strong inhibitory effects of niclosamide on the migration and invasion of DU145 cells, we further investigated its effect on the protein levels of MMP2, MMP9, cadherins and catenins. The results clearly demonstrated that niclosamide decreased protein levels of MMP2 and MMP9 in DU145 cells after a 24-hour treatment in a dose-dependent manner. As shown in Figure 2, niclosamide obviously decreased the protein level of MMP2 at a concentration as low as $0.2 \mu \mathrm{M}$, and $2.0 \mu \mathrm{m}$ of niclosamide induced nearly a complete loss of MMP2 expression. However, compared with the remarkable inhibition of MMP2, the effect of niclosamide on MMP9 seemed to be less potent, indicating that different mechanisms regulate the expression of these two proteins.

The results also clearly demonstrated that niclosamide potently altered the protein levels of cadherins and catenins in DU145 prostate cancer cells (Figure 2). A pan-cadherin antibody recognizing all members of the cadherin family was used to evaluate the effect of niclosamide on total cadherin expression. Generally, the protein level was dramatically decreased with niclosamide treatment. However, it is interesting to note that while treatment of niclosamide at higher doses (0.5-5.0 $\mu \mathrm{M})$ potently decreased protein levels, treatment at a lower dose $(0.2 \mu \mathrm{M})$ actually increased pan-cadherin expression. However, expression decreased with a dose of $0.5 \mu \mathrm{M}$ niclosamide and continued to decrease with increasing concentrations, until regaining expression at higher dosages of niclosamide $(\geq 2.0 \mu \mathrm{M})$. The result indicates that the

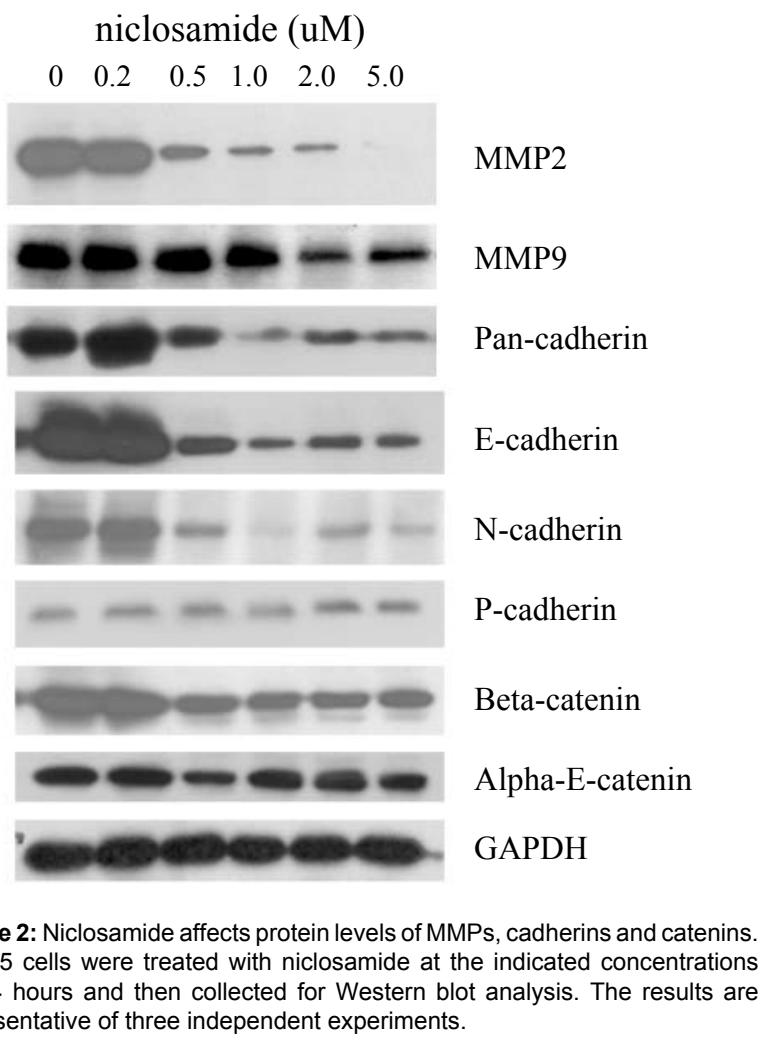

Figure 2: Niclosamide affects protein levels of MMPs, cadherins and catenins DU145 cells were treated with niclosamide at the indicated concentrations representative of three independent experiments. potent inhibition of cadherins by niclosamide might be due to its effects on individual cadherins. The antibodies against specific subgroups of cadherins were used for further Western blot analysis. Three classic cadherins, $E$-, $P$ - and $N$-cadherin, were differently affected by niclosamide. The protein level of $N$-cadherin (a mesenchymal marker) was dramatically inhibited by niclosamide as expected, but $E$-cadherin (an epithelial marker) was also potently suppressed by niclosamide. This finding might be explained by the controversial role of E-cadherin in the EMT process and in tumor progression in various contexts [33]. Compared to the potent inhibition on $\mathrm{E} / \mathrm{N}$-cadherin, niclosamide did not have a significant effect on the protein level of $P$-cadherin. Two key catenin members, $\beta$ - and $\alpha$-E-catenin were also altered by niclosamide treatment. The former was obviously suppressed by niclosamide, and the latter was not obviously changed. Compared with the potent effect of niclosamide on the cadherins, the effect of niclosamide on catenins is much milder, suggesting that niclosamide might interfere with EMT in DU145 cells mainly through regulation of the cadherins.

We have reported that niclosamide could potently inhibit the activation of STAT3 in DU145 cancer cells [21]. Given the important role of STAT3 in regulating cell motility and metastasis in prostate cancer, we postulated that the inhibitory effects of niclosamide on the STAT3 pathway might contribute greatly to the suppression of cell motility in DU145 cells.

DU145 cells harbor a high level of constitutively activated STAT3. To demonstrate if the inhibition by niclosamide on the motility of DU145 cells is dependent on STAT3 suppression, siRNA was used to knock down the level of STAT3 in DU145 cells. The wound healing assay was performed using cells transfected with either STAT3 SiRNA or a control scramble SiRNA (Scr-SiRNA). To avoid concealing an inhibitory effect of niclosamide on motility of DU145 cells, $25 \mathrm{nM}$ of STAT3 was utilized for transfection after preliminary optimization because this dose could obviously decrease the STAT3 protein level without significantly affecting the motility of DU145 cells. As shown in Figure 3B, Western blot analysis revealed efficient knockdown of STAT3 by STAT3 SiRNA compared with Scr-SiRNA. As expected, $2.0 \mu \mathrm{M}$ of niclosamide displayed significantly weaker inhibition of wound healing in DU145 cells transfected with STAT3 SiRNA than in those transfected with Scr-SiRNA (Figure 3A). The inhibitory effect of niclosamide was significantly decreased by $34.3 \%$ and $35.2 \%$ at 24 and 48 hours, respectively ( $\mathrm{P}<0.05$ for both) when comparing the STAT3 SiRNA group to the control Scr-SiRNA group (Figure 3C). This result strongly indicates that the effect of niclosamide on motility of DU145 cells is highly dependent on the STAT3 protein level in the cells.

Although we have demonstrated that STAT3 is involved in the inhibition by niclosamide on the motility of DU145 cells, suppression of STAT3 with siRNA only partly relieved the inhibitory activity of niclosamide. These data indicate that other signaling cascades might also be involved in the inhibition by niclosamide on the motility of DU145 cells. In addition to the STAT3 pathway, the MAPK pathway has been recently described to contribute to the development of metastatic PCA. Therefore, we evaluated the impact of niclosamide treatment on the MAPK pathway in DU145 cells.

Because Erk1/2 is a key component of the MAPK pathway and plays an important role in promoting cancer metastasis, the effect of niclosamide on the MAPK pathway was investigated by evaluating its impact on Erk1/2 activation in DU145 cells. As shown in Figure 4A, which is highly consistent with our previous observation, niclosamide significantly inhibited the activation of STAT3 but showed no obvious effect on Erk1/2 expression after a 4 hour treatment. However, when the treatment was extended to 24 hours, niclosamide exhibited strong 
A
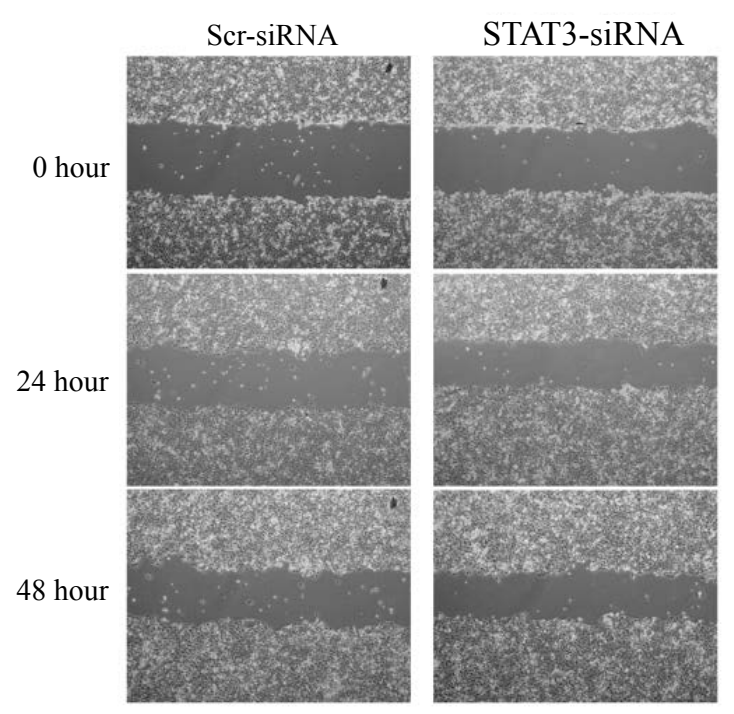

B

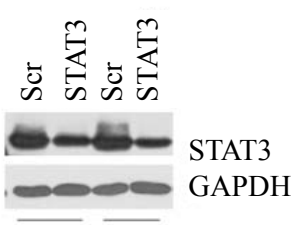

24 hour 48 hour
C

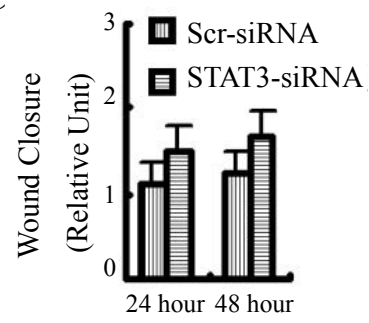

Figure 3: Inhibition of niclosamide on migration of DU145 cells is STAT3dependent. A: Blocking STAT3 decreases inhibitory effects of niclosamide on DU145 cell migration. DU145 cells were transfected with STAT3 siRNA or scrambled siRNA (Scr-SiRNA) and subjected to the wound healing assay. Cells were treated with $2 \mu \mathrm{M}$ of niclosamide, and images were taken at indicated time points after the wounds were created. B: Efficiency of siRNA interference shown by Western blot analysis. Cells were treated as in A and collected for Western blot analysis at indicated time points. C: Quantification of $A$. The results are representative of three independent experiments.

inhibition on the activation of Erk1/2, indicating that niclosamide might target upstream regulators of MAPK pathways and consequently affect Erk1/2 activation.

We further investigated if MAPK was involved in the inhibition by niclosamide on the motility of DU145 cells. The wound healing assay was carried out to observe effects of niclosamide on the wound closure with or without PD98059, which is a selective MAPK inhibitor. A concentration of $10.0 \mu \mathrm{M}$ PD98059 was chosen for the experiment because this dose induced an obvious inhibition of activation of Erk1/2 without significantly affecting wound closure. While niclosamide alone (at $1.0 \mu \mathrm{M})$ potently inhibited wound closure of DU145 cells by $63.5 \%$, its inhibition potency was significantly decreased to $43.2 \%$ when combined with PD98059 $(\mathrm{P}<0.01)$ (Figures $4 \mathrm{~B}$ and $4 \mathrm{C})$. This result revealed that down-regulating the MAPK pathway would weaken the inhibitory capacity of niclosamide on migration of DU145 cells, and it strongly indicates that the suppression of niclosamide on the MAPK pathway mediates its inhibitory effect on motility of the DU145 prostate cancer cell line.

In summary, we have successfully identified niclosamide as novel potent suppressor to inhibit the migration and invasion of DU145 prostate cancer cells. Further mechanism studies suggested that the suppression on STAT3 and MAPK pathways might be involved in

its strong inhibitory activities on migration and invasion of DU145. Niclosamide is a traditional anthelmintic drug that recently was demonstrated to have promising antitumor activity. Beside its inhibition on the STAT3 and MAPK pathway, its antitumor effect has been linked to its inhibition of other signaling pathways, such as the Notch [34], NF-kB [35], mTOR [36] and Wnt pathways [37]. Our results, combined with the knowledge that niclosamide suppresses many other signaling pathways involved in tumorigenesis and metastasis, leads us to conclude that niclosamide might serve as a potential candidate or a promsing lead compound for treating primary and metastatic prostate cancer.

\section{Experiments}

\section{Reagents and materials}

DU145 cells were purchased from ATCC (Manassas, USA) and were cultured in $\alpha$-MEM containing 10\% FBS unless otherwise indicated. Niclosamide was purified via recrystallization of the crude product (85\%) from ethanol, and the purity was over $99.0 \%$ (determined by

A

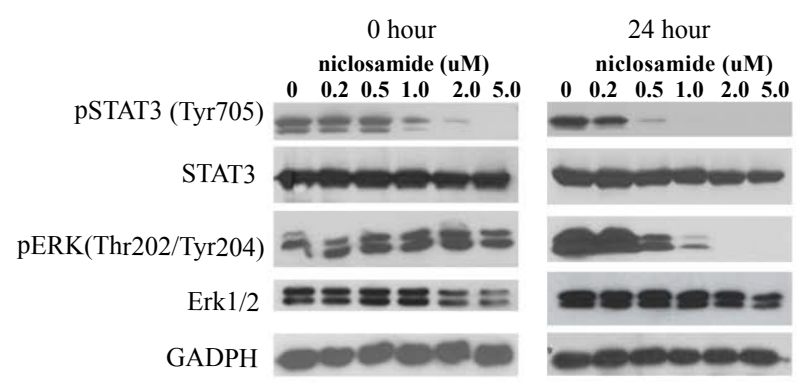

B

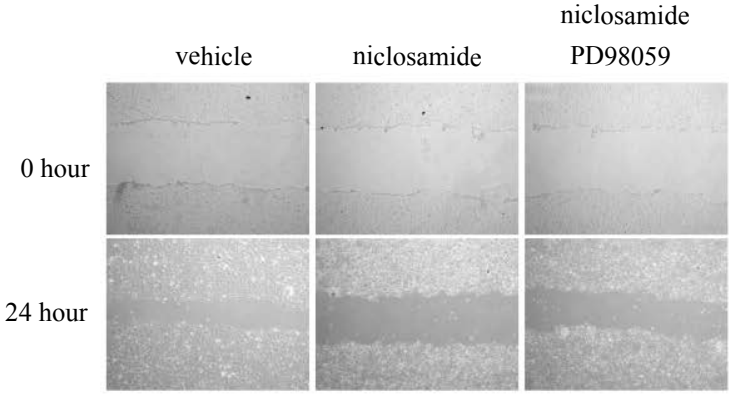

C

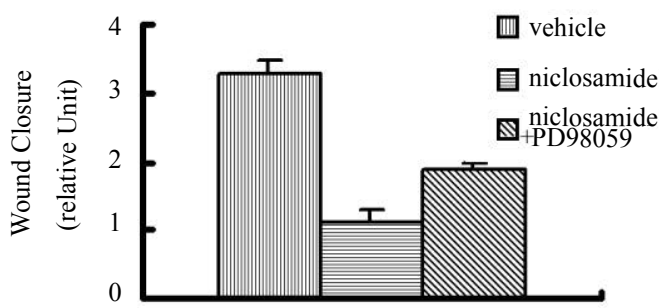

Figure 4: Inhibition of niclosamide on migration of DU145 cells is mediated by the MAPK signaling pathway. A: Elongated treatment with Niclosamide suppresses the MAPK pathway. DU145 cells were treated with niclosamide at the indicated concentrations for 4 hours (left panel) or 24 hours (right panel) and then collected for Western blot analysis. B: Blocking the MAPK pathway weakens the effect of niclosamide on the migration of DU145 cells. DU145 cells were treated with $2 \mu \mathrm{M}$ of niclosamide, $2 \mu \mathrm{M}$ of niclosamide combined with $10 \mu \mathrm{M}$ of PD98059, or vehicle control. Cells were then subjected to wound healing assay. Images were taken at the indicated time points after the wounds were created. C: Quantification of B. The results are representative of three independent experiments. 
high performance liquid chromatography). PD98059 was purchased from Sigma (Saint Louis, USA). Antibodies against STAT3, phosphorSTAT3(Tyr705), Erk1/2, phospho-Erk1/2 (Thr202/Tyr204), Pan-/ P-/ E-/ N-cadherin, $\beta / \alpha$-E-catenin were purchased from Cell Signaling Technology Inc. (CST, Danvers, USA). Antibodies against MMP2 and MMP9 were purchased from Abcam (Cambridge, UK).

\section{Wound healing/migration assay}

DU145 cells were plated in 6-well plates in complete medium $(2 \times 105$ cells/well $)$ that yielded a confluent monolayer one day after plating. The culture medium was then switched to low-serum containing medium ( $\alpha$-MEM with $1 \%$ FBS). The wound scratches were created with $200 \mu \mathrm{l}$ pipette tips, and the treatment was initiated. Wound closure was monitored at the indicated time points by photographing with a microscope at $40 \times$ magnification. Nine fields were monitored for each group. Wound closure was measured using the program ImagePro Plus and was estimated by the wound recovery distance calculated using the equation: Wound Closure $=($ Wound Gap Distance at $\mathrm{Tt}-$ Wound Gap Distance at T0).

\section{Boyden chamber/invasion assay}

Hanging cell culture inserts (Millipore, Billerica, USA) with an 8 $\mu \mathrm{m}$ polycarbonate filter membrane were pre-coated with extracellular matrix (ECM) (Sigma) and placed into 24-well plates. Complete medium containing the vehicle or indicated compounds were added into the lower chamber as a chemoattractant. DU145 cells were resuspended in serum-free medium containing the vehicle or indicated compounds and plated in the top chamber $(4 \times 104$ cells/well). At 24 hours after plating, the cells were fixed with $100 \%$ ice cold methanol and stained with $2 \%$ crystal violet (in $70 \%$ ethanol). Cells on the upper surface of the membrane were removed with a cotton swab, and the invading cells on the lower surface of the membrane were photographed with a microscope at $200 \times$ magnification. The cells were counted with the program Image J. Six random fields were quantified for each group.

\section{SiRNA interference}

STAT3 siRNA(STAT3-siRNA) and scrambled siRNA (Scr-siRNA) were purchased from RiboBio Ltd. (Guangzhou, China). DU145 cells were resuspended in complete medium $(1 \times 105 \mathrm{cells} / \mathrm{ml})$ and transfected with STAT3-siRNA or control Scr-siRNA duplexes (25 $\mathrm{pmol} / \mathrm{ml}$ ) using Lipofectamine 2000 (Invitrogen, Carlsbad, USA) according to the manufacturer's instructions. Transfected cells were transferred into 6-well plates for wound healing assays or into $2-\mathrm{cm}$ culture dishes for Western blot analysis.

\section{Western blot analysis}

Western blot analysis was carried out following the protocol outlined by Cell Signaling Technology Inc. as described previously [38].

\section{Statistics}

For the wound healing assay, the unpaired Student's $t$ test was used to assess significance between the groups. A $\mathrm{P}$ value of $\mathrm{P}<0.05$ was considered significant.

\section{Acknowledgments}

We thank the National Basic Research Program of China (\#2010CB529706, 2009CB940904), the National Natural Science Foundation (\#21072192), and the Key Project on Innovative Drug of Guangzhou City (grant \#2009Z1-E911, 2010JE551) for their financial support.

\section{References}

1. Jemal A, Siegel R, Xu J, Ward E (2010) Cancer statistics, 2010. CA Cancer J Clin 60: 277-300.
2. Jemal A, Siegel R, Ward E, Hao Y, Xu J, et al. (2009) Cancer statistics, 2009. CA Cancer J Clin 59: 225-249.

3. Salesi N, Carlini P, Ruggeri EM, Ferretti G, Bria E, et al. (2005) Prostate cancer the role of hormonal therapy. J Exp Clin Cancer Res 24: 175-180.

4. Sriprasad S, Feneley MR, Thompson PM (2009) History of prostate cancer treatment. Surg Oncol 18: 185-191.

5. Cooperberg MR, Lubeck DP, Meng MV, Mehta SS, Carroll PR (2004) The changing face of low-risk prostate cancer: trends in clinical presentation and primary management. J Clin Oncol 22: 2141-2149.

6. Arya M, Bott SR, Shergill IS, et al. (2006) Surg Oncol 151:17-128.

7. Sturge J, Caley MP, Waxman J (2011) Bone metastasis in prostate cancer: emerging therapeutic strategies. Nat Rev Clin Oncol 8: 357-368.

8. Yu H, Jove R (2004) The STATs of cancer--new molecular targets come of age. Nat Rev Cancer 4: 97-105.

9. Darnell JE (2005) Validating Stat3 in cancer therapy. Nat Med 11: 595-596.

10. Azare J, Leslie K, Al-Ahmadie H, Gerald W, Weinreb PH, et al. (2007) Constitutively activated Stat3 induces tumorigenesis and enhances cell motility of prostate epithelial cells through integrin beta 6 . Mol Cell Biol 27: 4444-4453.

11. Huang HF, Murphy TF, Shu P, Barton AB, Barton BE (2005) Stable expression of constitutively-activated STAT3 in benign prostatic epithelial cells changes their phenotype to that resembling malignant cells. Mol Cancer 4: 2

12. Ni Z, Lou W, Leman ES, Gao AC (2000) Inhibition of constitutively activated Stat3 signaling pathway suppresses growth of prostate cancer cells. Cancer Res 60: 1225-1228.

13. Barton BE, Karras JG, Murphy TF, Barton A, Huang HF (2004) Signal transducer and activator of transcription 3 (STAT3) activation in prostate cancer: Direct STAT3 inhibition induces apoptosis in prostate cancer lines. Mol Cancer Ther 3: 11-20.

14. Abdulghani J, Gu L, Dagvadorj A, Lutz J, Leiby B, et al. (2008) Stat3 promotes metastatic progression of prostate cancer. Am J Pathol 172: 1717-1728.

15. Gu L, Dagvadorj A, Lutz J, Leiby B, Bonuccelli G, et al. (2010) Transcription factor Stat3 stimulates metastatic behavior of human prostate cancer cells in vivo, whereas Stat5b has a preferential role in the promotion of prostate cancer cell viability and tumor growth. Am J Pathol 176: 1959-1972.

16. Sekine Y, Suzuki K, Remaley AT (2011) HDL and sphingosine-1-phosphate activate stat3 in prostate cancer DU145 cells via ERK1/2 and S1P receptors, and promote cell migration and invasion. Prostate 71: 690-699.

17. Royuela M, Arenas $\mathrm{MI}$, Bethencourt FR, SÃ (2002) Regulation of proliferation/apoptosis equilibrium by mitogen-activated protein kinases in normal, hyperplastic, and carcinomatous human prostate. Hum Pathol 33: 299-306

18. Uzgare AR, Kaplan PJ, Greenberg NM (2003) Differential expression and/or activation of P38MAPK, erk $1 / 2$, and jnk during the initiation and progression of prostate cancer. Prostate 55: 128-139.

19. Keller ET, Fu Z, Yeung K, Brennan M (2004) Raf kinase inhibitor protein: a prostate cancer metastasis suppressor gene. Cancer Lett 207: 131-137.

20. Junttila MR, Li SP, Westermarck J (2008) Phosphatase-mediated crosstalk between MAPK signaling pathways in the regulation of cell survival. FASEB J 22: 954-965

21. Ren X, Duan L, He Q, Zhang Z, Zhou Y, et al. (2010) Identification of Niclosamide as a New Small-Molecule Inhibitor of the STAT3 Signaling Pathway. ACS Med Chem Lett 1: 454-459.

22. Itoh $Y$, Nagase $H$ (2002) Matrix metalloproteinases in cancer. Essays Biochem 38: 21-36.

23. Roy R, Yang J, Moses MA (2009) Matrix metalloproteinases as novel biomarkers and potential therapeutic targets in human cancer. $\mathrm{J}$ Clin Oncol 27: 5287-5297.

24. Kessenbrock K, Plaks V, Werb Z (2010) Matrix metalloproteinases: regulators of the tumor microenvironment. Cell 141: 52-67.

25. Aimes RT, Quigley JP (1995) Matrix metalloproteinase-2 is an interstitial collagenase. Inhibitor-free enzyme catalyzes the cleavage of collagen fibrils and soluble native type I collagen generating the specific $3 / 4-$ and $1 / 4-$ length fragments. J Biol Chem 270: 5872-5876.

26. Fridman R, Toth M, Chvyrkova I, Meroueh SO, Mobashery S (2003) Cell 
Citation: Ren X, Zhang Z, Duan L, Ding K (2014) Identification of Niclosamide as a New Lead Compound to Suppress the Metastasis of Prostate Cancer Cells. Med chem 4: 511-516. doi:10.4172/2161-0444.1000187

surface association of matrix metalloproteinase-9 (gelatinase B). Cancer Metastasis Rev 22: 153-166.

27. Pellikainen JM, Ropponen KM, Kataja VV, Kellokoski JK, Eskelinen MJ, et al. (2004) Expression of matrix metalloproteinase (MMP)-2 and MMP-9 in breast cancer with a special reference to activator protein-2, HER2, and prognosis. Clin Cancer Res 10: 7621-7628.

28. Roomi MW, Monterrey JC, Kalinovsky T, Rath M, Niedzwiecki A (2009) Patterns of MMP-2 and MMP-9 expression in human cancer cell lines. Oncol Rep 21: 1323-1333.

29. Cowin P, Rowlands TM, Hatsell SJ (2005) Cadherins and catenins in breast cancer. Curr Opin Cell Biol 17: 499-508.

30. Bremnes RM, Veve R, Hirsch FR, Franklin WA (2002) The E-cadherin cell-cell adhesion complex and lung cancer invasion, metastasis, and prognosis. Lung Cancer 36: 115-124.

31. Hajra KM, Fearon ER (2002) Genes Chrosomes Cancer 34: 225-268.

32. Conacci-Sorrell M, Zhurinsky J, Ben-Ze'ev A (2002) The cadherin-catenin adhesion system in signaling and cancer. J Clin Invest 109: 987-991.
33. Rodriguez FJ, Lewis-Tuffin LJ, Anastasiadis PZ (2012) E-cadherin's dark side: possible role in tumor progression. Biochim Biophys Acta 1826: 23-31.

34. Wang AM, Ku HH, Liang YC, Chen YC, Hwu YM, et al. (2009) The autonomous notch signal pathway is activated by baicalin and baicalein but is suppressed by niclosamide in K562 cells. J Cell Biochem 106: 682-692.

35. Jin Y, Lu Z, Ding K, Li J, Du X, et al. (2010) Antineoplastic mechanisms of niclosamide in acute myelogenous leukemia stem cells: inactivation of the NFkappaB pathway and generation of reactive oxygen species. Cancer Res 70 : 2516-2527.

36. Balgi AD, Fonseca BD, Donohue E, Tsang TC, Lajoie P, et al. (2009) Screen for chemical modulators of autophagy reveals novel therapeutic inhibitors of mTORC1 signaling. PLoS One 4: e7124.

37. Chen M, Wang J, Lu J, Bond MC, Ren XR, et al. (2009) The anti-helminthic niclosamide inhibits Wnt/Frizzled1 signaling. Biochemistry 48: 10267-10274.

38. Ren X, Duan L, He Q, Zhang Z, Zhou Y (2010) Identification of Niclosamide as a New Small-Molecule Inhibitor of the STAT3 Signaling Pathway. ACS Med Chem Lett 1: 454-459. 